Global Journal of Energy and Environment
(ISSN: 2641-9947)

\title{
Power Planning
}

\author{
Peijun Jiang', Yiqian Gao', Ao Luo ${ }^{1}$
}

${ }^{1}$ School of Electrical and New Energy, China Three Gorges University 43002, China.

\begin{abstract}
This paper calculates the existing system reliability index and typical daily optimal load distribution, studies the optimization problem of the generator unit's investment plan in the next ten years, and uses the minimum cost of the investment plan to establish a multi-objective planning model. Combined with the *Correspondence to Author:

Peijun Jiang

School of Electrical and New Energy, China Three Gorges University artificial bee colony algorithm. It also discusses the impact of a large number of renewable energy power generation access power systems on traditional power system planning, and proposes an experimental model to solve this problem.

How to cite this article:

Based on the system enumeration method of fault enumeration, Peijun Jiang, Yiqian Gao, Ao Luo. enumerate all possible states of the system at each moment, consider the total cost of the system, increase the load demand constraint on the basis, and obtain the probability of occurrence of low-order faults. LOLP, EENS and power outage fees.

After the above analysis, after the fault order reaches 5th order, the LOLP of the system is negligible, so the power loss cost is considered to be 4th order. Using the minimum total cost as the objective function, the artificial bee colony algorithm is used to find the optimal solution.

Power Planning. Global Journal of Energy and Environment, 2019,1:7.

The uncertainty of the output of renewable energy in the power system greatly increases the risk of operation scheduling, does not guarantee the quality of power, and is currently economically

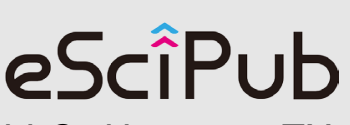

eSciPub LLC, Houston, TX USA. Website: https://escipub.com/
\end{abstract}


poor. In view of the difficulties caused by the system components in solving nonlinear constrained power supply planning, an experimental model is also established.

Keywords: Mixed integer programming; System enumeration method; Artificial bee colony algorithm; Cost calculation; Optimization design

\section{Restatement of the problem}

Power system planning can be divided into power supply planning, transmission network planning, and distribution network planning. The rationality of power supply planning directly affects the reliability, economy and power quality of system operation. Power planning is used to seek a most economical power development solution based on load forecasting for a certain period of time, while meeting the technical indicators of the power system.

According to the IEEE-RTS system unit technical and economic parameters, typical daily load and unit reliability parameters given in the appendix, mathematical models and algorithms are established to solve the following problems: 1. If only the unit investment cost and operating cost, the total cost of the power system, and the reliability index of the existing system LOLP, EENS, and power outage loss are calculated. On the basis of the second, optimize the $2030 \mathrm{crew}$ installation plan. Consider the impact of highorder system failures and reliability index con- straints in the cost of power outages separately, and plan the annual unit installation plan for the next 10 years.

2. Explain the impact on the planning of traditional power systems when a large number of new energy sources are connected to the power system, and discuss the solution to the problem of power supply planning for solving nonlinear constraints in light of the actual situation.

Second, the model hypothesis

Hypothesis 1: The output power of the generator is instantaneously variable at the beginning of any period of time and does not take time, and the change process has no cost.

Hypothesis 2: The output power of the generator is stable and does not interfere with other natural factors.

Hypothesis 3: The impact of the failure of the new unit on the original unit is not considered.

Hypothesis 4: Ignore the impact of system faults above the fifth order when calculating the reliability performance index.

\section{2. the Symbol Description}

\begin{tabular}{cl}
\hline Variable symbol & \multicolumn{1}{c}{ Symbol Description } \\
\hline$i$ & Generator type \\
$j$ & Number of generators \\
$t$ & Future years \\
$p_{i}$ & Investment cost of a single type of generator \\
$F_{t}$ & Equivalent investment cost of a single generator \\
$A$ & in the coming year \\
$r$ & Equal annual value \\
$J_{i(1,2,3,4)}$ & discount rate \\
\hline
\end{tabular}


Peijun Jiang et al., GJEE, 2019; $1: 7$

\begin{tabular}{cl}
\hline$k_{i(1,2,3,4)}$ & Number of units put into operation \\
$M$ & Additional unit capacity \\
$N$ & Actually commissioned unit capacity \\
$L$ & 2030 crew investment costs \\
$a_{i}$ & Power generation cost factor for the first type of \\
& unit $a$ \\
$b_{i}$ & Power generation cost factor for the first type of \\
& unit $b$ \\
$c_{i}$ & Power generation cost factor for the first type of \\
$\tau$ & unit $c$ \\
\hline
\end{tabular}

\section{3. the Analysis of the Problem}

\subsection{Analysis of Problem One}

It is necessary to consider the unit investment cost and operating cost, and calculate the optimal load distribution plan for each unit in the 12th and 24th hour of the typical day of the existing system.

(1) Calculate the optimal load distribution plan for a certain period of the typical system on a typical day. There is no additional installation unit, no need to consider the unit investment cost, only consider the operation cost, and use the running cost as the objective function to establish a single-objective nonlinear programming model. When considering the optimal load distribution scheme, since the number of generators of each type is limited, and the output power of a single unit should be between the minimum unit output and the unit capacity, and the output power of the unit must meet the load demand, convert it. As a constraint.

It is necessary to consider both the unit investment cost and the system operation cost. It is known that the daily load standard value in 2030 is the same as the typical day, and the 2030 installation plan is optimized.

Because the unit investment cost and system operation cost should be considered, the sum of the two costs is used as the objective function to establish a multi-objective planning model. Under the premise of meeting the technical indicators of the power system, the solution when the objective function value is minimized is the optimization scheme. . The difference is that the load demand is not simply to meet the total load demand of the day, but to meet the load demand for each time period of the day.

Calculate the reliability index of the existing system on the basis of typical daily load, and on this basis, consider the total cost to optimize the 2030 crew installation plan.

If the unit power loss is known, calculate the reliability index LOLP, EENS and power outage loss of the existing system. According to the failure rate and repair rate of the original given in the appendix, calculate the probability that the components are in normal operation state and fault operation state respectively. It is necessary to enumerate all possible states of the system at each moment based on the system enumeration method of fault enumeration. get the probability of a heavy fault.

The problem needs to plan for the additional installed capacity plan for each of the next decade, with a known peak load growth rate of $3 \%$ for the next decade.

(1) It is necessary to consider the influence of high-order system fault state as much as possi- 
ble in the blackout loss fee. The objective function and constraint condition of this problem are similar to problem four (2), that is, the minimum total cost is used as the objective function to calculate the optimal scheme. It has been analyzed in question 4 that after the fault order reaches 5th order, the LOLP of the system is negligible, so the power loss cost here takes into account the 4th order, but because the exhaustive algorithm is complicated, it is difficult to obtain the result. This problem is intended to be solved using the artificial bee colony algorithm.

(2) This problem does not need to consider the cost of power outage loss, that is, the objective function is the minimum value of unit investment cost and operating cost. The constraint conditions only need to consider the reliability index and load demand, and establish a two-objective nonlinear programming model to solve.

\subsection{Analysis of Problem}

For the second problem, through the literature review, combined with the characteristics of renewable energy generator sets, the possible impact on power system planning will be explained. Due to the large number of actual power system components, it will have a great impact on soIving nonlinear constrained power supply planning. An unconstrained function filterless method for solving nonlinear constrained optimization problems is established, and an experimental model is established.

\section{4. the Establishment and Solution of the Pro- blem Model}

\subsection{Establishment and Solution of Annually Added Unit Model}

\subsubsection{Establishment of the annual add-on un- it model}

This question requires us to plan the type and quantity of additional units installed in each of the next 10 years, that is, to determine the type and quantity of installed units in each of the next 10 years in order. We change the model of question four to get the corresponding solution model. Since 2020-2030, the annual peak load growth rate is $3 \%$, that is, considering the thirdorder failure when calculating the cost of power outage loss, the objective function is:

$$
\min H=\sum_{\tau=1}^{10} A+C R F \cdot C_{t}+C_{L t}
$$

In the formula, $A$ is the annual value such as investment cost, the annual value such as operating cost, and the cost of power outage loss. The constraint conditions of this problem are the same as the second problem, namely: the maximum number of operating units in the year, the upper limit of the total installed capacity, the balance of power balance, the capacity reserve constraint, the N-1 criterion, the output power constraint of a single unit, and the load demand constraint.

In summary, the multi-objective nonlinear programming model is:

$$
\begin{gathered}
\min H=\sum_{\tau=1}^{10} A+C R F \cdot C_{t}+C_{L t} \\
\text { s.t. }\left\{\begin{array}{l}
0 \leq k_{1} \leq 1,0 \leq k_{2} \leq 2,0 \leq k_{3} \leq 3,0 \leq k_{4} \leq 2 \\
J_{1} \leq 3, \quad J_{2} \leq 6, \quad J_{3} \leq 10, \quad J_{4} \leq 10 \\
J_{i} \in N, i=1,2,3,4 \\
k_{i} \leq J_{i}, i=1,2,3,4 \\
N+3405 \geq 2850 \times 1.3 \\
M+3405 \geq 2850 \times 1.3 \times 1.2 \\
3405-400+N \geq 1.3 \times 2850 \\
d_{i \min } \leq d_{i} \leq d_{i \max }, i=1,2,3,4 \\
L_{\tau}+3405=1.3 \times 2850 e_{\tau}
\end{array}\right.
\end{gathered}
$$

\subsubsection{Solving the annual model of the install- ed unit}

Since the variables in the above model are too difficult to solve using the exhaustive method, the artificial bee colony algorithm is used to sol- 
ve the model. $x_{i d}^{\prime}=x_{i d}+\varphi_{i d}\left(x_{i d}-x_{k d}\right) \varphi_{i d} \in(-1,1)$ The artificial bee colony algorithm is briefly introduced below.

The Artificial bee colony algorithm:

$$
p_{i}=\frac{f i t_{i}}{\sum_{i=1}^{S N} f i t_{i}}
$$

The artificial bee colony algorithm is a global optimization algorithm based on group intelligence. The whole algorithm is divided into three operations: employment bee, observation bee and $d$ etection bee. The goal is to find the source of the most nectar.

Hire bee: use the previous honey source information to find a new honey source and share the honey source information with the observation bee;

Observing bees: Waiting in the hive and looking for new sources of honey based on information shared by the hiring bees;

Scouting Bees: Looking for a new and valuable source of honey, they randomly search for honey sources near the hive.

Figure 3: Artificial Bee Colony Algorithm Flowchart

Assuming that the spatial solution is dimensional, the employment bee is based on updating the honey source information, which refers to the honey source, and at the same time determines the amount of honey nectar; the observation bee uses the information provided by the employment bee to calculate the probability of each honey source using a certain adaptation function. The probability formula is :

$$
x_{i d}=x_{d}^{\min }+r\left(x_{d}^{\max }-x_{d}^{\min }\right)
$$

In the formula, the number of employed bees is the fitness value of the possible solution. Then use roulette to select a honey source. For the selected honey source, observe the bee to search for a new possible solution according to the above probability formula, and determine the amount of nectar of the honey source. The detection bee is used to determine whether a honey source is raised within a specified step. If it is not raised, the honey source is discarded, and a honey source is reinitialized and searched again. The scout bee searches for new possible solutions by the following formula.

Among them, are the random numbers on the interval, and are the lower and upper bounds of the dimension.

The model is solved according to the artificial bee colony algorithm:

Table 12: Additions for the next decade

\begin{tabular}{ccccc}
\hline Ynit & Unit 1 & Unit 2 & Unit 3 & Unit 4 \\
\hline 2020 & 1 & 2 & 0 & 1 \\
2021 & 0 & 1 & 0 & 1 \\
2022 & 0 & 1 & 0 & 0 \\
2023 & 0 & 0 & 1 & 0 \\
2024 & 0 & 1 & 1 & 0 \\
2025 & 0 & 1 & 0 & 1 \\
2026 & 0 & 0 & 0 & 2 \\
2027 & 0 & 0 & 1 & 0 \\
2028 & 0 & 0 & 1 & 1 \\
2023 & 0 & 1 & 0 & 0 \\
\hline
\end{tabular}




$\begin{array}{lllll}2030 & 0 & 0 & 1 & 1\end{array}$

\subsubsection{Annually installed unit model}

(1) Analysis of results

In order to ensure the lowest cost and the stan-

dby rate every year, the relationship between output power and load power balance should be satisfied. In the case of a certain load power, it is necessary to use a generator with a large capacity and a low investment cost as much as possible. According to the parameters of known generators. The generator chosen for the optimal solution fully meets this requirement.

(2) Model evaluation

Problem 5 uses the system enumeration method of the fault enumeration method to obtain the corresponding system state to obtain the corresponding power failure loss cost and the objective function value.

From the analysis of the meaning of the problem, the higher the order, the higher the accuracy of the calculation, but the longer the calculation takes time and the lower the calculation efficiency. Only by setting the appropriate order can the calculation accuracy and efficiency be relatively higher. In the problem of the fourth question, by analyzing and processing the corresponding data, the following conclusions are $\mathrm{dr}$ awn: after the fault order reaches 5 th order, the LOLP of the system is negligible, so the order is set to $1-5$ steps, so that the conclusion More accurate and persuasive.

For the description of the method: Problem 5 uses the improved artificial bee colony algorithm to intelligently optimize the model, which greatly reduces the control parameters and calculation amount, improves the calculation efficiency, and saves time under the premise of ensuring the accuracy of the results.

\subsection{Establishment and Solution of Annually Added Unit Model}

\subsubsection{Establishment of the annual add-on un-}

\section{it model}

This question requires us to plan the type and quantity of installed units in each of the next 10 years. Different from model (1), there is no need to consider the cost of power outage loss, that is, the objective function is the minimum of the sum of the unit's investment cost and operating cost. The function is:

$$
\min L=\sum_{\tau=1}^{10} A+C R F \cdot C_{t}
$$

In the formula, $A$ is the annual value such as investment cost, and $C R F \cdot C_{t}$ is the annual value such as operating cost.

The constraints of this problem only need to consider the load demand constraints and the reliability index constraints.

Therefore, the dual-objective nonlinear programming model established is:

$$
\begin{gathered}
\min L=\sum_{\tau=1}^{10} A+C R F \cdot C_{t} \\
\text { s.t. }\left\{\begin{array}{l}
L O L P \leq 0.5 \% \\
\sum_{i=1}^{32} Q_{i \tau}<1.03 \times 2850 e_{\tau}
\end{array}\right.
\end{gathered}
$$

among them $L O L P=\sum_{a \in S} P_{k}^{a}$

\subsubsection{Solution of the annual installed unit $\mathrm{m}$ - odel}

The solution for the next ten years can be obtained by solving the two-objective nonlinear equations: 
Peijun Jiang et al., GJEE, 2019; 1:7

\begin{tabular}{ccccc}
\hline $\begin{array}{c}\text { Model } \\
\text { Years }\end{array}$ & Unit 1 & Unit 2 & Unit 3 & Unit 4 \\
\hline 2020 & 2 & 0 & 1 & 0 \\
2021 & 0 & 0 & 2 & 1 \\
2022 & 0 & 1 & 0 & 1 \\
2023 & 0 & 0 & 2 & 0 \\
2024 & 1 & 0 & 0 & 2 \\
2025 & 0 & 1 & 0 & 0 \\
2026 & 1 & 0 & 0 & 0 \\
2027 & 0 & 1 & 0 & 0 \\
2028 & 0 & 2 & 0 & 1 \\
2029 & 0 & 0 & 1 & 2 \\
2030 & 0 & 1 & 1 & 0 \\
\hline
\end{tabular}

4.2.3 analysis of the results of the annual in-

\section{stalled unit model (2)}

To ensure the lowest cost, only load demand constraints and reliability index constraints are considered. In the case of a certain load power, the accounting group with large capacity, low investment cost and low operating cost should be selected as much as possible. According to the parameters of the known unit, the generator selected for the optimal solution fully meets this requirement.

\section{Model Evaluation}

\subsection{Advantages of the model}

(1) The comparison of the annual value is converted into the annual equivalent investment cost, which simplifies the calculation.

(2) The first question is to use the system enumeration method of fault enumeration method to obtain the corresponding state, and then intelligently optimize the model according to the artificial bee colony algorithm, which greatly reduces the control parameters and calculation amount, improves the calculation efficiency, and guarantees the result. Significant savings in time under the premise of accuracy.

(3) According to the analysis of the actual situation and the requirements of the topic, the calculation order is controlled within a reasonable range, which simplifies the calculation.

\section{2 the Shortcomings of the model}

(1) can be reduced, but the problem of excessive calculation cannot be avoided.

(2) The time effect of adding additional units and units in the year of 2030 was not considered, resulting in imprecise models.

Seven, the improvement and promotion of the model

In the process of model establishment and problem solving, we found that increasing the time effect when considering the types and number of additional units in 2030 can more fully reflect the process of optimizing the allocation, thus improving the accuracy of calculation and the persuasiveness of the model.

So we have built the following improved model:

The installed capacity is:

$$
M=250 J_{1}+100 J_{2}+65 J_{3}+50 J_{4}
$$

Commissioned capacity

$$
N=250 K_{1}+100 K_{2}+65 K_{3}+50 K_{4}
$$

Actual operating unit capacity at the first hour of the day

$$
L_{\tau}=\sum_{i=1}^{4} Q_{i \tau}
$$




\section{Operating costs}

$$
C_{t}=365 \sum_{\tau=0}^{24} a_{i} Q_{i \tau}{ }^{2}+b_{i} Q_{i \tau}+c_{i}
$$

Under the conditions of satisfying the maximum number of dispatching units, the integer constraint of the unit's incremental load, the single output power constraint of the generator, and the balance of the power balance, the operation plan is calculated year by year according to the model, and the commissioning plan for the year of 2030 is derived. Happening.

In the model of question five, we did not consider the impact of the new unit on the original unit and the cost of power outage caused by its own operation, which should be better considered in the improved model.

The improved model is more suitable for the actual situation and is more in line with the meaning of the question. At the same time, the interaction between variables is considered and quantified, which makes the model richer and more universal, and can adapt to the optimal scheduling problem under different conditions.

\section{Reference}

1. Gao Yajing, Zhou Ming, Li Gengyin, Li Rui, Xiao Limin. Calculation of available transmission capacity based on Markov chain and fault enumeration method[J]. Proceedings of the CSEE,2006 (13): 41-46.

2. Zuo Siran, Wang Zhongyu, Fan Wenbo, Fu Jinwei, Guan Shilei. An Improved State Estimation Method for Artificial Bee Colony Distribution Network[J]. Electric Measurement \& Instrumentation, 2018, 55(24): 40-45.

3. Zuo Shuangyong, Wang Xiangling, Zhu Zhibin. A Method for Solving Non-Discriminant Function Filterless Problems for Nonlinear Constrained Optimization Problems[J].Mathematics and Practice

4. Xiang Yue, Liu Junyong, Wei Zhenbo, Cao Yinli, 